\title{
A New Version of the Accelerated Overrelaxation Iterative Method
}

\author{
Shi-Liang Wu and Yu-Jun Liu \\ School of Mathematics and Statistics, Anyang Normal University, Anyang 455000, China \\ Correspondence should be addressed to Shi-Liang Wu; wushiliang1999@126.com
}

Received 22 May 2014; Revised 8 July 2014; Accepted 9 July 2014; Published 26 August 2014

Academic Editor: Shuqian Shen

Copyright (C) 2014 S.-L. Wu and Y.-J. Liu. This is an open access article distributed under the Creative Commons Attribution License, which permits unrestricted use, distribution, and reproduction in any medium, provided the original work is properly cited.

\begin{abstract}
Hadjidimos (1978) proposed a classical accelerated overrelaxation (AOR) iterative method to solve the system of linear equations, and discussed its convergence under the conditions that the coefficient matrices are irreducible diagonal dominant, $L$-matrices, and consistently orders matrices. In this paper, a new version of the AOR method is presented. Some convergence results are derived when the coefficient matrices are irreducible diagonal dominant, $H$-matrices, symmetric positive definite matrices, and $L$-matrices. A relational graph for the new AOR method and the original AOR method is presented. Finally, a numerical example is presented to illustrate the efficiency of the proposed method.
\end{abstract}

\section{Introduction}

Consider the following linear system:

$$
A x=b,
$$

where $A \in \mathbb{R}^{n \times n}, b \in \mathbb{R}^{n}$ are given and $x \in \mathbb{R}^{n}$ is unknown. System of form (1) appears in many applications such as linear elasticity, fluid dynamics, and constrained quadratic programming [1-4]. When the coefficient matrix of the linear system (1) is large and sparse, iterative methods are recommended against direct methods. In order to solve (1) more effectively by using the iterative methods, usually, efficient splittings of the coefficient matrix $A$ are required. For example, the classical Jacobi and Gauss-Seidel iterations are obtained by splitting the matrix $A$ into its diagonal and offdiagonal parts.

For the numerical solution of (1), the accelerated overrelaxation (AOR) method was introduced by Hadjidimos in [5] and is a two-parameter generalization of the successive overrelaxation (SOR) method. In certain cases the AOR method has better convergence rate than Jacobi, JOR, GaussSeidel, or SOR method $[5,6]$. Sufficient conditions for the convergence of the AOR method have been considered by many authors including [6-14]. To improve the convergence rate of the AOR method, the preconditioned AOR (PAOR) method has been considered by many authors including [15-21]. Although Krylov subspace methods [4, 22] are considered as one kind of the important and efficient iterative techniques for solving the large sparse linear systems because these methods are cheap to be implemented and are able to fully exploit the sparsity of the coefficient matrix, Krylov subspace methods are very slow or even fail to converge when the coefficient matrix of (1) is often extremely ill-conditioned and highly indefinite.

The purpose of this paper is to present a new version of the accelerated overrelaxation (AOR) method for the linear system (1), which is called the quasi accelerated overrelaxation (QAOR) method. We discuss some sufficient conditions for the convergence of the QAOR method when the coefficient matrices are irreducible diagonal dominant, $H$-matrices, symmetric positive definite matrices, and $L$ matrices.

The remainder of the paper is organized as follows. In Section 2 the QAOR method is derived. In Section 3, some convergence results are given for the QAOR method when the coefficient matrices are irreducible diagonal dominant, $H$-matrices, symmetric positive definite matrices, and $L$ matrices. A relational graph for $\mathrm{QAOR}$ and $\mathrm{AOR}$ is presented 
in Section 4. Finally, in Section 5 a numerical example is presented to illustrate the efficiency of the proposed method.

\section{The QAOR Method}

To introduce the QAOR method, firstly, a brief review of the classical AOR method is required.

For any splitting, $A=M-N$ with $\operatorname{det}(M) \neq 0$, the basic iterative method for solving (1) is

$$
x^{(i+1)}=M^{-1} N x^{(i)}+M^{-1} b, \quad i=0,1, \ldots
$$

Let

$$
A=D-A_{L}-A_{U}
$$

where $D$ is a nonsingular diagonal matrix and $A_{L}$ and $A_{U}$ are strictly lower and upper triangular matrices, respectively. Then the classical AOR method in [5] is defined:

$$
\begin{array}{r}
\left(D-r A_{L}\right) x^{(i+1)}=\left[(1-\omega) D+(\omega-r) A_{L}+\omega A_{U}\right] x^{(i)}+\omega b, \\
i=1,2, \ldots,
\end{array}
$$

where $r$ is an acceleration parameter and $\omega$ is an overrelaxation parameter. Its iterative matrix is

$$
\begin{aligned}
L_{r, \omega} & =\left(D-r A_{L}\right)^{-1}\left[(1-\omega) D+(\omega-r) A_{L}+\omega A_{U}\right] \\
& =(I-r L)^{-1}[(1-\omega) I+(\omega-r) L+\omega U],
\end{aligned}
$$

where $L=D^{-1} A_{L}$ and $U=D^{-1} A_{U}$. Obviously, the iterative matrix of the Jacobi method is $L_{0,1}$, the iterative matrix of the Gauss-Seidel method is $L_{1,1}$, and the iterative matrix of the successive overrelaxation (SOR) method is $L_{\omega, \omega}$.

In fact, if we introduce matrices

$$
\begin{aligned}
& M_{1}=D-r A_{L}, \\
& N_{1}=(1-\omega) D+(\omega-r) A_{L}+\omega A_{U},
\end{aligned}
$$

then

$$
A=\frac{1}{\omega}\left(M_{1}-N_{1}\right), \quad L_{r, \omega}=M_{1}^{-1} N_{1} .
$$

Therefore, one can readily verify that the AOR method can be induced by the matrix splitting $A=(1 / \omega)\left(M_{1}-N_{1}\right)$.

To establish the QAOR method, we consider the following matrix splitting of the coefficient matrix $A$; that is to say,

$$
\begin{aligned}
& M_{2}=(1+\omega) D-r A_{L} \\
& N_{2}=D+(\omega-r) A_{L}+\omega A_{U} .
\end{aligned}
$$

Then

$$
A=\frac{1}{\omega}\left(M_{2}-N_{2}\right)
$$

Based on the above matrix splitting (8), the QAOR method is defined as follows:

$$
\begin{aligned}
& \left((1+\omega) D-r A_{L}\right) x^{(i+1)} \\
& \quad=\left[D+(\omega-r) A_{L}+\omega A_{U}\right] x^{(i)}+\omega b, \quad i=1,2, \ldots,
\end{aligned}
$$

and its iterative matrix is

$$
\begin{aligned}
Q_{r, \omega} & =\left((1+\omega) D-r A_{L}\right)^{-1}\left[D+(\omega-r) A_{L}+\omega A_{U}\right] \\
& =((1+\omega) I-r L)^{-1}[I+(\omega-r) L+\omega U] .
\end{aligned}
$$

Comparing the QAOR method with the AOR method, it is easy to see that the iteration matrix of the QAOR method is similar to that of the AOR method. Based on this fact, the QAOR method may conserve all the advantages of the AOR method. If $\omega=r$, the QAOR reduces to the QSOR method. The QSOR method is called the KSOR method as well [23, 24].

Next, we will discuss some sufficient conditions for the convergence of the QAOR method when the coefficient matrices are irreducible diagonal dominant, $H$-matrices, symmetric positive definite matrices, and $L$-matrices.

\section{Main Results}

When $A$ is an irreducible matrix with weak diagonal dominance, obviously, both the coefficient matrix $A$ and the corresponding diagonal matrix $D$ are nonsingular. Based on this case, we have the following theorem for the QAOR method.

Theorem 1. If $A$ is an irreducible matrix with weak diagonal dominance, then the QAOR method converges for all $-1 \leq r \leq$ 1 and $\omega>0$.

Proof. We assume that for the eigenvalue $\lambda$ of $Q_{r, \omega}$ we have $|\lambda| \geq 1$. For this eigenvalue the relationship below holds:

$$
\operatorname{det}\left(Q_{r, \omega}-\lambda I\right)=0 .
$$

By performing a simple series of transformations, we have

$$
\operatorname{det}(W)=0,
$$

where

$$
W=I-\frac{r(\lambda-1)+\omega}{\lambda-1+\lambda \omega} L-\frac{\omega}{\lambda-1+\lambda \omega} U .
$$

The coefficients of $L$ and $U$ in (14) are less than one in modulus. To prove this it is sufficient and necessary to prove that

$$
\begin{aligned}
& |\lambda-1+\lambda \omega| \geq|r(\lambda-1)+\omega|, \\
& |\lambda-1+\lambda \omega| \geq|\omega| .
\end{aligned}
$$

If $\lambda^{-1}=q e^{i \theta}$ where $q$ and $\theta$ are real with $0<q \leq 1$, then the first inequality in (15) is equivalent to

$$
\begin{aligned}
& \left(1-r^{2}\right)\left(1+q^{2}\right)-\left(\left(1-r^{2}\right)+(1+r) \omega\right) 2 q \cos \theta \\
& +2 \omega+\omega^{2}-\omega^{2} q^{2}+2 r \omega q^{2} \geq 0,
\end{aligned}
$$


which holds for $r=-1$ (in this case, obviously, $\omega\left(1-q^{2}\right)(2+$ $\omega) \geq 0$ ). Since $\left(1-r^{2}\right)+(1+r) \omega \geq 0,(16)$ holds for all real $\theta$ if and only if it holds for $\cos \theta=1$. Thus, (16) is equivalent to

$$
\begin{aligned}
& \left(1-r^{2}\right)(1-q)^{2} \\
& +\omega\left[\omega\left(1-q^{2}\right)+2(1-q)(1-r q)\right] \geq 0,
\end{aligned}
$$

which is true. The second inequality in (15) is equivalent to

$$
(1+\omega)^{2}-2 q(1+\omega) \cos \theta+q^{2}-\omega^{2} q^{2} \geq 0,
$$

which, for the same reason, must be satisfied for $\cos \theta=1$. Thus, we have

$$
(1+\omega)^{2}+q\left(1+\omega^{2}\right) \geq 0
$$

which is also true. That is, for all $-1 \leq r \leq 1$ and $\omega>0, W$ is nonsingular which contradicts with $\operatorname{det}(W)=0$. Therefore, $\rho\left(Q_{r, \omega}\right)<1$.

When $A=D-A_{L}-A_{U}$ is an $H$-matrix, it follows that $\rho(|B|)<1$ with

$$
B=D^{-1}\left(A_{L}+A_{U}\right)=L+U .
$$

Theorem 2. If $A$ is an $H$-matrix and $0 \leq r \leq \omega \quad(\omega \neq 0)$, then the QAOR method converges.

Proof. Let $Q=((1+\omega) I-r|L|)$. Then

$$
\begin{aligned}
& \left|((1+\omega) I-r L)^{-1}\right| \\
& =\frac{1}{1+\omega}\left|\left(I-\frac{r}{1+\omega} L\right)^{-1}\right| \\
& =\frac{1}{1+\omega}\left|I+\frac{r}{1+\omega} L+\frac{r^{2}}{(1+\omega)^{2}} L^{2}+\cdots+\frac{r^{n-1}}{(1+\omega)^{n-1}} L^{n-1}\right| \\
& \leq \frac{1}{1+\omega}\left(I+\frac{r}{1+\omega}|L|+\frac{r^{2}}{(1+\omega)^{2}}|L|^{2}\right. \\
& \left.\quad+\cdots+\frac{r^{n-1}}{(1+\omega)^{n-1}}|L|^{n-1}\right) \\
& =((1+\omega) I-r|L|)^{-1}=Q^{-1} .
\end{aligned}
$$

Let $R=I+(\omega-r)|L|+\omega|U|$. Then

$$
|I+(\omega-r) L+\omega U| \leq I+(\omega-r)|L|+\omega|U| .
$$

Obviously, we have

$$
\begin{aligned}
\left|Q_{r, \omega}\right|= & \mid((1+\omega) I-r L)^{-1} \\
& \times(I+(\omega-r) L+\omega U) \mid \leq Q^{-1} R,
\end{aligned}
$$

which implies

$$
\rho\left(Q_{r \omega}\right) \leq \rho\left(Q^{-1} R\right)
$$

$\rho\left(Q^{-1} R\right)<1$ if and only if

$$
\begin{aligned}
Q-R= & (1+\omega) I-r|L| \\
& -(I+(\omega-r)|L|+\omega|U|)=\omega(I-|B|)
\end{aligned}
$$

is a monotone matrix. Since $A$ is an $H$-matrix, then $\omega(I-|B|)$ is a monotone matrix. Therefore it is completed.

Let

$$
\begin{aligned}
& \bar{Q}=\frac{1}{\omega}\left((1+\omega) D-r A_{L}\right), \\
& \bar{R}=\frac{1}{\omega}\left(D+(\omega-r) A_{L}+\omega A_{U}\right) .
\end{aligned}
$$

When $A=D-A_{L}-A_{U}$ is symmetric positive definite, obviously, $\bar{Q}$ is nonsingular. It is easy to see that

$$
A=\bar{Q}-\bar{R}, \quad Q_{r, \omega}=\bar{Q}^{-1} \bar{R} .
$$

In this case, the QAOR method converges if $M=\bar{Q}+\bar{Q}^{T}-A$ is positive definite [2]. By the simple computations, we have

$$
M=\frac{2+\omega}{\omega} D+\frac{\omega-r}{\omega}\left(A_{L}+A_{U}\right)
$$

That is to say, the QAOR method converges if

$$
D^{-1 / 2} M D^{-1 / 2}=\frac{2+\omega}{\omega} I+\frac{\omega-r}{\omega} D^{-1 / 2}\left(A_{L}+A_{U}\right) D^{-1 / 2}
$$

is positive definite. Let $\mu_{i}(i=1,2, \ldots, n)$ be eigenvalues of $D^{-1 / 2}\left(A_{L}+A_{U}\right) D^{-1 / 2}$. The left in (29) is positive definite if and only if

$$
\frac{2+\omega}{\omega}+\frac{\omega-r}{\omega} \mu_{i}>0, \quad i=1,2, \ldots, n
$$

Since $D^{-1 / 2}\left(A_{L}+A_{U}\right) D^{-1 / 2}$ and $D^{-1}\left(A_{L}+A_{U}\right)=B$ are similar, then both have the same eigenvalues. Let $\mu=\min _{i} \mu_{i}$. If the following inequality is satisfied

$$
(2+\omega)+(\omega-r) \mu>0, \quad \text { for } \omega>0,
$$

then the QAOR method converges. Therefore, we have the following theorem.

Theorem 3. Assume that $A=D-A_{L}-A_{U}$ is symmetric positive definite. Let $\mu_{i}(i=1,2, \ldots, n)$ be eigenvalues of $B=$ $D^{-1}\left(A_{L}+A_{U}\right), \mu=\min _{i} \mu_{i}$, and $0 \leq r \leq \omega(\omega \neq 0)$. If

$$
(2+\omega)+(\omega-r) \mu>0,
$$

then the QAOR method converges.

When $A=D-A_{L}-A_{U}$ is an $L$-matrix, the following theorem is derived.

Theorem 4. If $A$ is an L-matrix and $0 \leq r \leq \omega \quad(\omega \neq 0)$, then the QAOR method converges for $\rho\left(L_{0,1}\right)<1$. 
Proof. Assume that $\lambda=\rho\left(Q_{r, \omega}\right) \geq 1$. Based on our assumptions, we easily get that

$$
\begin{aligned}
I+(\omega-r) L+\omega U \geq 0 & \\
((1+\omega) I-r L)^{-1}= & \frac{1}{1+\omega}\left(I-\frac{r}{1+\omega} L\right)^{-1} \\
= & \frac{1}{1+\omega}\left[I+\frac{r}{1+\omega} L+\frac{r^{2}}{(1+\omega)^{2}} L^{2}\right. \\
& \left.+\cdots+\frac{r^{n-1}}{(1+\omega)^{n-1}} L^{n-1}\right]
\end{aligned}
$$

$\geq 0$.

Thus, for the iteration matrix $Q_{r, \omega}$ we have

$$
Q_{r, \omega}=((1+\omega) I-r L)^{-1}[I+(\omega-r) L+\omega U] \geq 0 .
$$

That is, $Q_{r, \omega}$ is a nonnegative matrix. If $x \neq 0$ is the corresponding eigenvector, we have

$$
Q_{r, \omega} x=\lambda x
$$

which is equivalent to

$$
\left(\frac{\omega-r+r \lambda}{\omega} L+U\right) x=\frac{\lambda-1+\lambda \omega}{\omega} x
$$

From (37), we have

$$
\frac{\lambda-1+\lambda \omega}{\omega} \leq \rho\left(\frac{\omega-r+r \lambda}{\omega} L+U\right) .
$$

Obviously, $(\omega-r+r \lambda) / \omega \geq 1$. Therefore,

$$
\begin{aligned}
0 & \leq \frac{\omega-r+r \lambda}{\omega} L+U \leq \frac{\omega-r+r \lambda}{\omega}(L+U) \\
& =\frac{\omega-r+r \lambda}{\omega} L_{0,1} .
\end{aligned}
$$

Combining (38) with (39), we have

$$
\lambda-1+\lambda \omega \leq(\omega-r+r \lambda) \rho\left(L_{0,1}\right) .
$$

By simple manipulation, we have

$$
\rho\left(L_{0,1}\right) \geq \frac{\lambda-1+\lambda \omega}{\omega-r+r \lambda} \geq 1 .
$$

If $\rho\left(L_{0,1}\right)<1$, then

$$
\frac{\lambda-1+\lambda \omega}{\omega-r+r \lambda}<1
$$

which implies $\lambda<1$ so that if $\rho\left(L_{0,1}\right)<1$ then so does the QAOR method.

Further, we have the following theorem.
Theorem 5. Let $A=D-A_{L}-A_{U}$ be an L-matrix and $\lambda=$ $\rho\left(Q_{r, \omega}\right)$. If $0 \leq r \leq \omega(\omega \neq 0)$, then

$$
\frac{\lambda-1+\lambda \omega}{\omega}=\rho\left(\frac{\omega-r+r \lambda}{\omega} L+U\right) .
$$

Proof. Based on Theorem 4, obviously, here it is need to prove

$$
\frac{\lambda-1+\lambda \omega}{\omega} \geq \rho\left(\frac{\omega-r+r \lambda}{\omega} L+U\right) .
$$

Let $\rho(((\omega-r+r \lambda) / \omega) L+U)=a$. There exists a nonzero vector $y \geq 0$ such that

$$
\left[\frac{\omega-r+r \lambda}{\omega} L+U\right] y=a y,
$$

which is equivalent to

$$
\begin{aligned}
& \left((1+\omega) I-\frac{\lambda(1+\omega) r}{1+\omega a} L\right)^{-1} \\
& \quad \times[I+(\omega-r) L+\omega U] y=\frac{\omega a+1}{1+\omega} y .
\end{aligned}
$$

Let

$$
T=\left((1+\omega) I-\frac{\lambda(1+\omega) r}{1+\omega a} L\right)^{-1}[I+(\omega-r) L+\omega U] .
$$

Obviously, $(\omega a+1) /(1+\omega) \leq \rho(T)$. Since $\lambda-1+\lambda \omega \leq \omega a$, we have

$$
\left((1+\omega) I-\frac{\lambda(1+\omega) r}{1+\omega a} L\right)^{-1} \leq((1+\omega) I-r L)^{-1},
$$

which implies $T \leq Q_{r, \omega}$. Therefore, we have

$$
\frac{\omega a+1}{1+\omega} \leq \rho(T) \leq \rho\left(Q_{r, \omega}\right)=\lambda .
$$

That is to say,

$$
a \leq \frac{\lambda-1+\lambda \omega}{\omega}
$$

which is completed.

Some remarks on (43) are given as follows.

(i) Obviously, $\lambda \geq 1 /(1+\omega)$. If $\lambda=1$, then $\rho(B)=1$.

(ii) If $\lambda=1 /(1+\omega)$, then $\rho(B)=0$. In fact, we have

$$
\begin{aligned}
0 & =\rho\left(\frac{\omega-r+r(1 /(1+\omega))}{\omega} L+U\right) \\
& =\rho\left(\left(1-\frac{r}{1+\omega}\right) L+U\right) \\
& \geq\left(1-\frac{r}{1+\omega}\right) \rho(B) .
\end{aligned}
$$

(iii) $0<\rho(B)<1$ if and only if $1 /(1+\omega)<\lambda<1$. In this case, from (43) we have

$$
\frac{(\omega-r) \rho(B)+1}{1+\omega-r \rho(B)} \leq \lambda \leq \frac{\omega \rho(B)+1}{1+\omega} .
$$

(iv) $\rho(B)>1$ if and only if $\lambda>1$. 
TABLE 1: $\rho$, IT, and RES of QAOR.

\begin{tabular}{lccccc}
\hline$n$ & $w$ & $r$ & $\rho$ & IT & CPU \\
\hline 8 & 0.9 & 0.6 & 0.575 & 63 & 0.015 \\
16 & 0.95 & 0.2 & 0.7188 & 102 & 0.056 \\
20 & 0.9 & 0.3 & 0.8068 & 155 & 0.084 \\
25 & 0.8 & 0.4 & 0.9198 & 385 & 0.156 \\
\hline
\end{tabular}

TABLE 2: $\rho$, IT, and RES of QSOR.

\begin{tabular}{lcccc}
\hline$n$ & $w$ & $\rho$ & IT & CPU \\
\hline 8 & 0.9 & 0.5726 & 62 & 0.015 \\
16 & 0.95 & 0.6977 & 94 & 0.035 \\
20 & 0.9 & 0.789 & 141 & 0.076 \\
25 & 0.8 & 0.9123 & 352 & 0.098 \\
\hline
\end{tabular}

\section{A Relational Graph for QAOR and AOR}

Based on the above discussion, we have

$$
\begin{aligned}
Q_{r, \omega}= & \left((1+\omega) D-r A_{L}\right)^{-1}\left[D+(\omega-r) A_{L}+\omega A_{U}\right] \\
= & ((1+\omega) I-r L)^{-1}[I+(\omega-r) L+\omega U] \\
= & \left(I-\frac{r}{1+\omega} L\right)^{-1}\left[\frac{1}{1+\omega} I+\frac{\omega-r}{1+\omega} L+\frac{\omega}{1+\omega} U\right] \\
= & \left(I-\frac{r}{1+\omega} L\right)^{-1} \\
& \times\left[\left(1-\frac{\omega}{1+\omega}\right) I+\frac{\omega-r}{1+\omega} L+\frac{\omega}{1+\omega} U\right] .
\end{aligned}
$$

Let $s=\omega /(1+\omega)$ and $t=r /(1+\omega)$. Therefore, we have

$$
Q_{r, \omega}=(I-t L)^{-1}[(1-s) I+(s-t) L+s U]=L_{t, s} .
$$

That is to say, when $s=\omega /(1+\omega)$ and $t=r /(1+\omega)$, the QAOR method reduces to the AOR method. Based on this case, Figure 1 describes the relationship between the QAOR method and the AOR method.

\section{Numerical Example}

Now let us consider the following example to assess the feasibility and effectiveness of the QAOR iteration method. Suppose that $b=A e\left(e=(1,1, \ldots, 1)^{T}\right)$ and the coefficient matrix $A$ of (1) is given by

$$
A= \begin{cases}a_{i j}=\frac{1}{10 j}-\frac{1}{20}, & i>j, \\ a_{i i}=1, & 1 \\ a_{i j}=\frac{1}{10(i-j)}-\frac{1}{20}, & i<j .\end{cases}
$$

The initial guess for all tests is zero. The tests are performed in MATLAB 7.0. In Tables 1 and 2, we list the value of the spectral radius $\rho$ of iterative matrix, the iteration

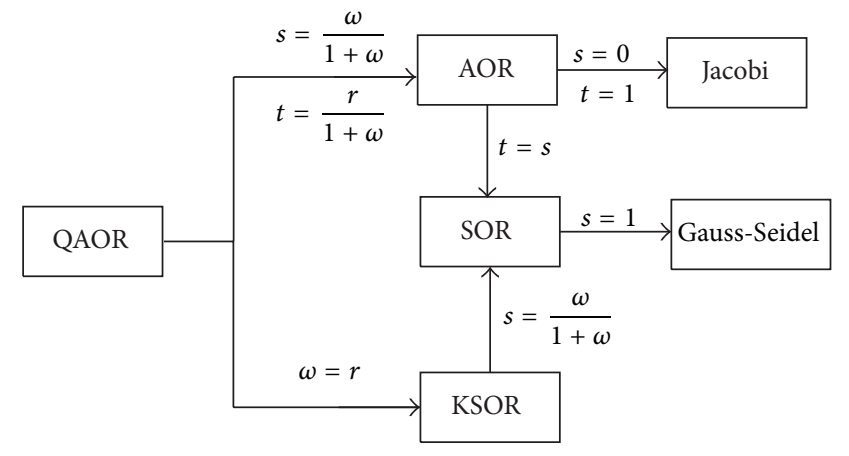

FIgURE 1: A graph for QAOR and AOR.

numbers (IT), and CPU's time (CPU) with the different value of $w$ and $r$ when the QAOR (QSOR) iteration is used to solve the linear system (1).

From Tables 1 and 2, the iteration numbers and CPU's time of QSOR are less than those of QAOR. That is to say, the QAOR iteration is not much better than the QSOR iteration under certain conditions.

\section{Conflict of Interests}

The authors declare that there is no conflict of interests regarding the publication of this paper.

\section{Acknowledgments}

This research was supported by NSFC (no. 11301009), by Science \& Technology Development Plan of Henan Province (no. 122300410316), and in part by the Natural Science Foundations of Henan Province (no. 13A110022).

\section{References}

[1] R. S. Varga, Matrix Iterative Analysis, Springer, Berlin, Germany, 2000.

[2] D. M. Young, Iterative Solution of Large Linear Systems, Academic Press, New York, NY, USA, 1971.

[3] A. Berman and R. J. Plemons, Nonnegative Matrices in the Mathematics Sciences, SIAM, Philadelphia, Pa, USA, 1994.

[4] Y. Saad, Iterative Methods for Sparse Linear Systems, PWS Publishing, Boston, Mass, USA, 1996.

[5] A. Hadjidimos, "Accelerated overrelaxation method," Mathematics of Computation, vol. 32, no. 141, pp. 149-157, 1978.

[6] G. Avdelas and A. Hadjidimos, "Some theoretical and computational results concerning the accelerated overrelaxation (AOR) method," L'Analyse Numérique et la Théorie de L'approximation, vol. 9, no. 1, pp. 5-10, 1980.

[7] A. K. Yeyios, "A necessary condition for the convergence of the accelerated overrelaxation (AOR) method," Journal of Computational and Applied Mathematics, vol. 26, no. 3, pp. 371373, 1989.

[8] Z. Bai, "The monotone convergence rate of the parallel nonlinear AOR method," Computers \& Mathematics with Applications, vol. 31, no. 7, pp. 1-8, 1996. 
[9] Z.-Z. Bai, "Parallel nonlinear AOR method and its convergence," Computers \& Mathematics with Applications, vol. 31, no. 2, pp. 21-31, 1996.

[10] Z. Bai, "Asynchronous multisplitting AOR methods for a class of systems of weakly nonlinear equations," Applied Mathematics and Computation, vol. 98, no. 1, pp. 49-59, 1999.

[11] L. Cvetković and V. Kostić, "A note on the convergence of the AOR method," Applied Mathematics and Computation, vol. 194, no. 2, pp. 394-399, 2007.

[12] Z. X. Gao and T. Z. Huang, "Convergence of AOR method," Applied Mathematics and Computation, vol. 176, no. 1, pp. 134140, 2006.

[13] J.-Y. Yuan and X.-Q. Jin, "Convergence of the generalized AOR method," Applied Mathematics and Computation, vol. 99, no. 1, pp. 35-46, 1999.

[14] W. Li and W.-W. Sun, "Comparison results for parallel multisplitting methods with applications to AOR methods," Linear Algebra and Its Applications, vol. 331, no. 1-3, pp. 131-144, 2001.

[15] J. H. Yun, "Comparison results of the preconditioned AOR methods for L-matrices," Applied Mathematics and Computation, vol. 218, no. 7, pp. 3399-3413, 2011.

[16] H.-J. Wang and Y.-T. Li, "A new preconditioned AOR iterative method for L-matrices," Journal of Computational and Applied Mathematics, vol. 229, no. 1, pp. 47-53, 2009.

[17] S. Wu and T. Huang, "A modified AOR-type iterative method for L-matrix linear systems," The ANZIAM Journal, vol. 49, no. 2, pp. 281-292, 2007.

[18] Y.-T. Li, C.-X. Li, and S.-L. Wu, "Improvements of preconditioned AOR iterative method for L-matrices," Journal of Computational and Applied Mathematics, vol. 206, no. 2, pp. 656-665, 2007.

[19] Y.-T. Li, C.-X. Li, and S.-L. Wu, "Improving AOR method for consistent linear systems," Applied Mathematics and Computation, vol. 186, no. 1, pp. 379-388, 2007.

[20] L. Wang and Y.-Z. Song, "Preconditioned AOR iterative methods for M-matrices," Journal of Computational and Applied Mathematics, vol. 226, no. 1, pp. 114-124, 2009.

[21] M. Wu, L. Wang, and Y. Song, "Preconditioned AOR iterative method for linear systems," Applied Numerical Mathematics, vol. 57, no. 5-7, pp. 672-685, 2007.

[22] Z. Bai, "Sharp error bounds of some Krylov subspace methods for non-Hermitian linear systems," Applied Mathematics and Computation, vol. 109, no. 2-3, pp. 273-285, 2000.

[23] I. K. Youssef, "On the successive overrelaxation method," Journal of Mathematics and Statistics, vol. 8, no. 2, pp. 176-184, 2012.

[24] I. K. Youssef and A. A. Taha, "On the modified successive overrelaxation method," Applied Mathematics and Computation, vol. 219, no. 9, pp. 4601-4613, 2013. 


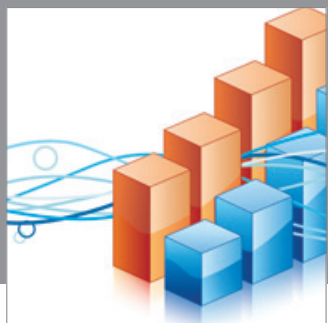

Advances in

Operations Research

mansans

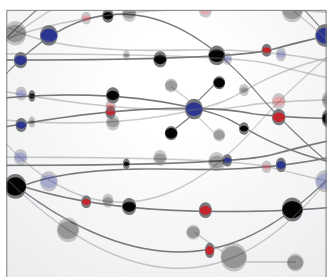

The Scientific World Journal
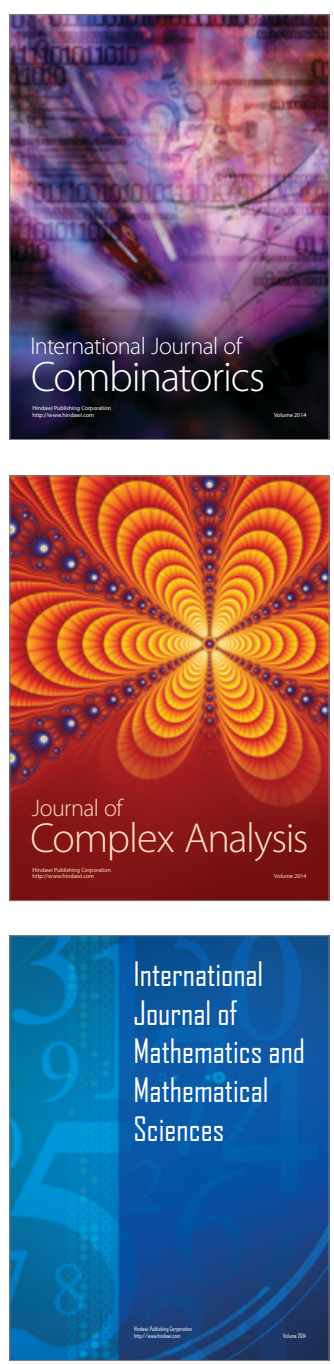
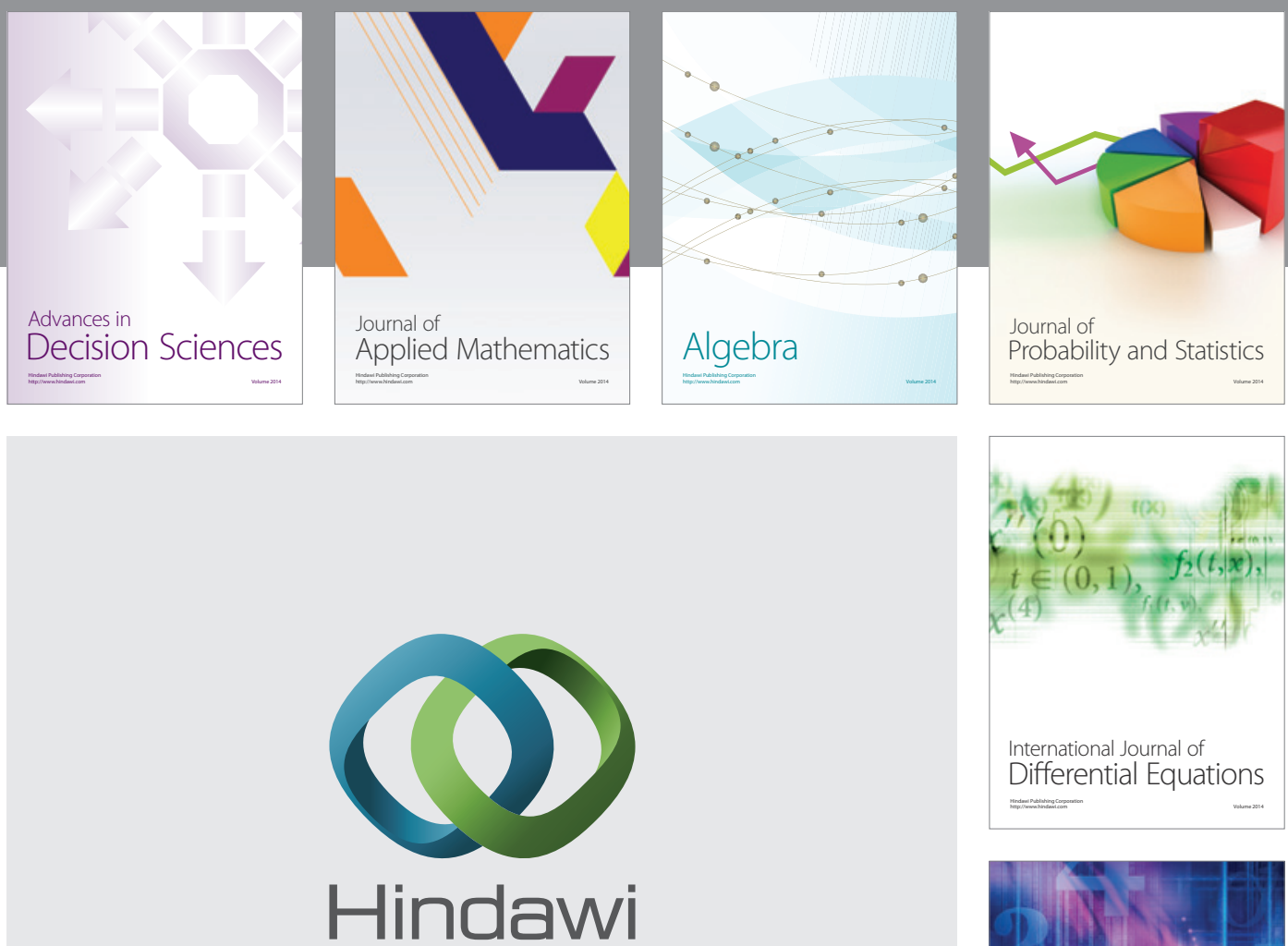

Submit your manuscripts at http://www.hindawi.com
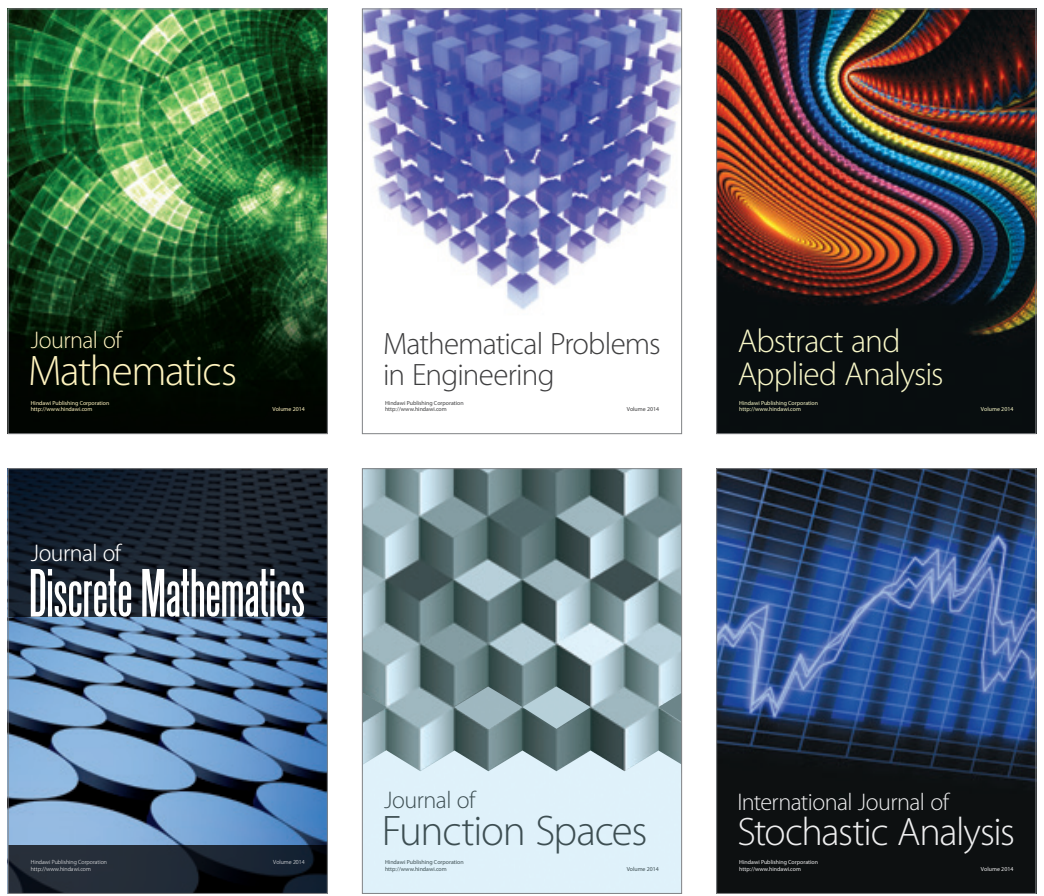

Journal of

Function Spaces

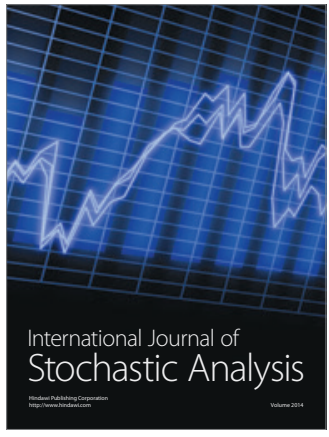

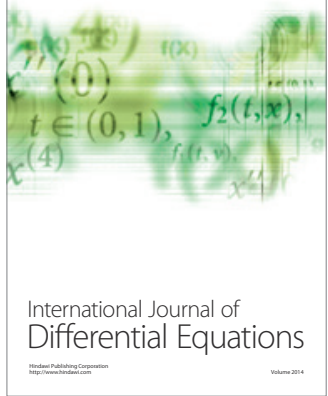
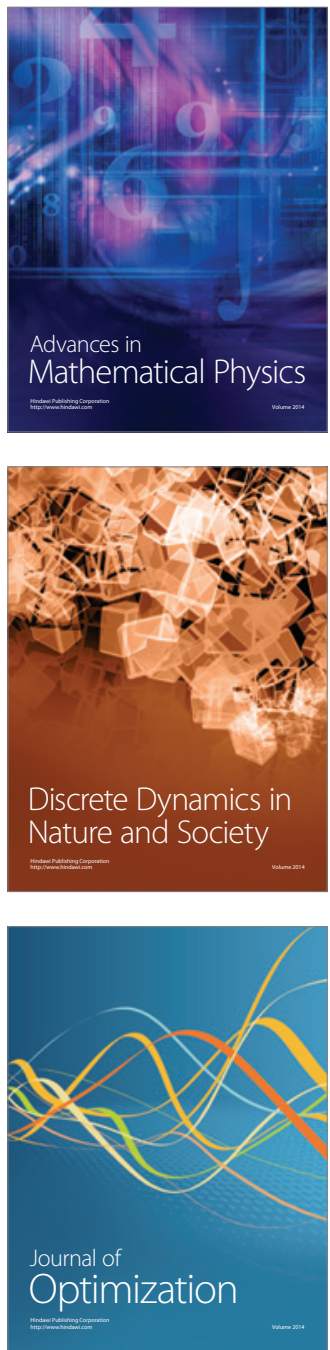